\title{
Discovery of Entity Synonym Using Anchor Text and URLs
}

\author{
Mamta Kathuria ${ }^{1}$, Anurahda $\operatorname{Singh}^{2}$, C. K. Nagpal ${ }^{3}$ and Neelam Duhan ${ }^{4}$ \\ ${ }^{1}$ Assistant Professor, YMCA University of Science \& Technology, \\ Faridabad (India) \\ ${ }^{2}$ Student (M.Tech), YMCA University of Science \& Technology, \\ Faridabad (India) \\ ${ }^{3}$ Professor, YMCA University of Science \& Technology, Faridabad (India) \\ ${ }^{4}$ Assistant Professor, YMCA University of Science \& Technology, \\ Faridabad (India) \\ ${ }^{1}$ mamtakathuria@ymcaust.ac.in, ${ }^{2}$ anuradhasngh13@gmail.com, \\ ${ }^{3}$ nagpalckumar@rediffmail.com, ${ }^{4}$ neelam.duhan@gmail.com
}

\begin{abstract}
In the current scenario, the web queries have become more and more pin-pointed so as to find results relating to specific entity in a specific context of time, place, etc. For example, information pertaining to a movie-show, a particular train, newspaper of a particular date, performance of a particular stock etc. All these references associated with a particular entity are known as entity references. The problem with these references is that they vary with the heterogeneous contexts of the web and one may not be getting the required answers to his/her query owing to these varied entity references known as entity synonyms. These entity synonyms cannot be handled through lexical resources like WordNet [1]. Therefore, every search engine will have to create its own mechanism for finding the entity synonyms of a particular entity in order to properly answer the users' queries, the process being known as entity resolution. In recent past, many researchers have tried to devise the mechanisms to generate the entity synonyms. This paper is also an effort in this direction and creates a rich set of entity synonyms for a given entity using inbound anchor text and URLs.
\end{abstract}

Keywords: Entity, Candidate Entity Synonym, Web Query, Inbound Anchor text, Entity Synonym Extraction

\section{Introduction}

With the growth of the web, users have been making diverse forms of queries relating to a variety of domains concerned with daily life issues. These queries associated with products, brands, recipes, weather forecast, show timings, quotes for various products etc. are being searched by common users to accomplish their daily needs. These searches related to entities can be best sorted out from the latest product catalogs and associated databases if the references are specific. However, if the references are general and refer to common entities, then product catalogs and databases may not be available. When the entities are well known, the synonyms can be found by the usage of sources like Wikipedia [2] and FreeBase [3]. For instance, the Bhabha Atomic research Center may be referred as Bhabha Institute, BARC, Atomic Energy Center, and Nuclear Energy Center etc. However, for the common entities these online resources may not work.

The problem with these generic entities is their multiple types of references to the same entity due to different creators of the web pages. For example, a paper like The Hindustan Times may be referred as The HT, HT, The Hindustan, The Hindustan Times Today, and

Received (May 15, 2017), Review Result (August 31, 2017), Accepted (September 20, 2017) 
Hindustan Times etc. The movie Bahubali-The Conclusion is also referred to as Bahubali2. Thus, there is a requirement to devise a mechanism to find entity synonyms for the common entities to enable the search engines to provide a rich search experience to its users.

The best possibility to find various varieties of entity synonyms available on the web is through the exploration of the web and to find the set of possible candidate references which can be further pruned. The paper considers such search efforts carried out in the recent past and proposes a novel technique that creates a rich set of entity synonyms. The proposed work will help in improving search relevance, enrich users' experience, query auto-suggestion, creation of entity dictionary, recommendation system and e-discovery etc.

The rest of the paper is organized as follows: Section 2 contains the formal description of the concepts used in this paper. Section 3 talks about web based empirical methods available in the literature to find out the entity synonym set for a given input string. Section 4 defines problem and objectives set for the work. Section 5 contains details of proposed methodology for entity resolution that provides better and enhanced results compared to its predecessors. Section 6 discusses the usage of results. Section 7 talks about the conclusion and future scope of the work.

\section{Basic Terminologies}

Entity: An entity refers to a place, person, thing, event or abstraction having a distinct and separate existence from other instances of similar attributes. The reference to the entity may be local or global depending upon the context of the underlying domain.

Entity Identifier: Formal nomenclature for the entity e.g. The Times of India, The Hindustan Times, Kabhi-Kabhi, Dilwale Dulhaniya Le Jayenge, i20, Santro Xing etc.

Entity Synonyms: A list of formal and informal identifiers referring to the same entity i.e commonly used alternative name references to describe the entity under consideration e.g. TOI and Times of India refer to the same entity. In the same way, Tere Bin Laden-2 and Tere Bin Laden dead or alive are not different.

To mathematically define the concept of entity synonyms, we take the help of the following concepts:

S: Universal set of strings over an alphabet

E: Universal set of entities

$E_{X}$ : A list of entities over the domain $X$ for example $E_{\text {Movies }}$ will be a set of entities over the movie domain.

Now we can define a function $\mathrm{F}$ having two arguments. The first one being an arbitrary string $s \varepsilon S$ and the second one being the entity domain $E_{X}$. Then the function $F\left(s, E_{X}\right)->e$ $\mathrm{E}$ maps the string $\mathrm{s}$ to a single or a set of entities in the global domain $\mathrm{E}$ which is a superset of Ex , thereby making a local reference as global.

Entity Synonym: Two strings s1 and s2 defined over the set $\mathrm{S}$ are said to be entity synonyms iff $\mathrm{F}\left(\mathrm{s} 1, \mathrm{E}_{\mathrm{X}}\right)=\mathrm{F}\left(\mathrm{s} 2, \mathrm{E}_{\mathrm{X}}\right)$

Entity Hyponym: A string s1 is a entity hyponym of the string s2 (both defined over the set $S)$ iff $F\left(s 1, E_{X}\right) F\left(s 2, E_{X}\right)$. 
Entity Hypernym: A string s1 is a entity hypernym of the string s2 (both defined over the set $S)$ iff $F\left(s 1, E_{X}\right) F\left(s 2, E_{X}\right)$.

The problem of finding the entity synonyms of a string $\mathrm{s}$ can be mathematically described as a situation to create a set Ws of strings w's such as:

$\mathrm{Ws}=\left\{\mathrm{w} \in \mathrm{S} \mid \mathrm{F}\left(\mathrm{s}, \mathrm{E}_{\mathrm{X}}\right)=\mathrm{F}\left(\mathrm{w}, \mathrm{E}_{\mathrm{X}}\right)\right\}$

The set $\mathrm{W}=\{\mathrm{w} 1, \mathrm{w} 2 \ldots \ldots \mathrm{wk}\}$ contains entity synonyms for the string $\mathrm{s}$ over the domain $\mathrm{X}$. Given a string s over the domain $\mathrm{X}$, we have to find out $\mathrm{W}$ in the context of Ex.

The subsequent section talks about such empirical methods as used by the various researchers along with their merits and demerits.

\section{Literature Survey}

With the time, growth of the web round the globe has made it a universally accepted information resource. This vast ocean of knowledge contains wide variety of references to the each entity, made by heterogeneous types of content creators and the web searchers. These heterogeneous references, if not properly taken care of, shall lead to inadequate supply of information to the web searcher despite the availability of the information. A need, therefore, has been long felt to cover this gap by grouping the references leading to same common entity into a set known as the set of entity synonyms and the process is referred to as entity resolution. The primitive approach in this regard has been the use of abbreviations, acronyms, Wikipedia and Freebase references. All these mechanisms have not been able to cover the web as a whole and its heterogeneity. Thus only solution for creating these set of references (entity synonyms) must include the use of web-ocean as source and fishing out the references. The literature of past ten years contains many such efforts. This section covers to these efforts and discusses their relative merits and demerits.

Pinky Paul et al., [4] have presented a study about entity search engine. They have discussed the basic architecture of entity search engine as shown in Figure 1. The approach is static and involves the possible basic modules of an Entity Search Engine. These modules accomplish the task of entity candidate extraction from the web, categorization, assignment of relevance and ranking. The architecture is quite general and provides an overview of the process.

The different approaches used in the entity resolution may differ in the method of entity extraction process which may be offline or online. The result computation can also be based upon some intermediate approach like pseudo document or reference table etc. The synonym generation process can also be static or dynamic. We take up different approaches in the above context.

Surajit Chaudhuri et al., [5] have proposed a method to find correlation between a candidate string and an entity in order to overcome the shortcomings of string-based similarity measures that does not reflect the common knowledge that users generally provide for the candidate string in the question. They proposed new document-based similarity measures to calculate the similarity in the context of many documents containing the candidate string. The main disadvantage of their approach is that they have performed computations on a small set of relevant documents containing the given candidate string to measure the correlation. 


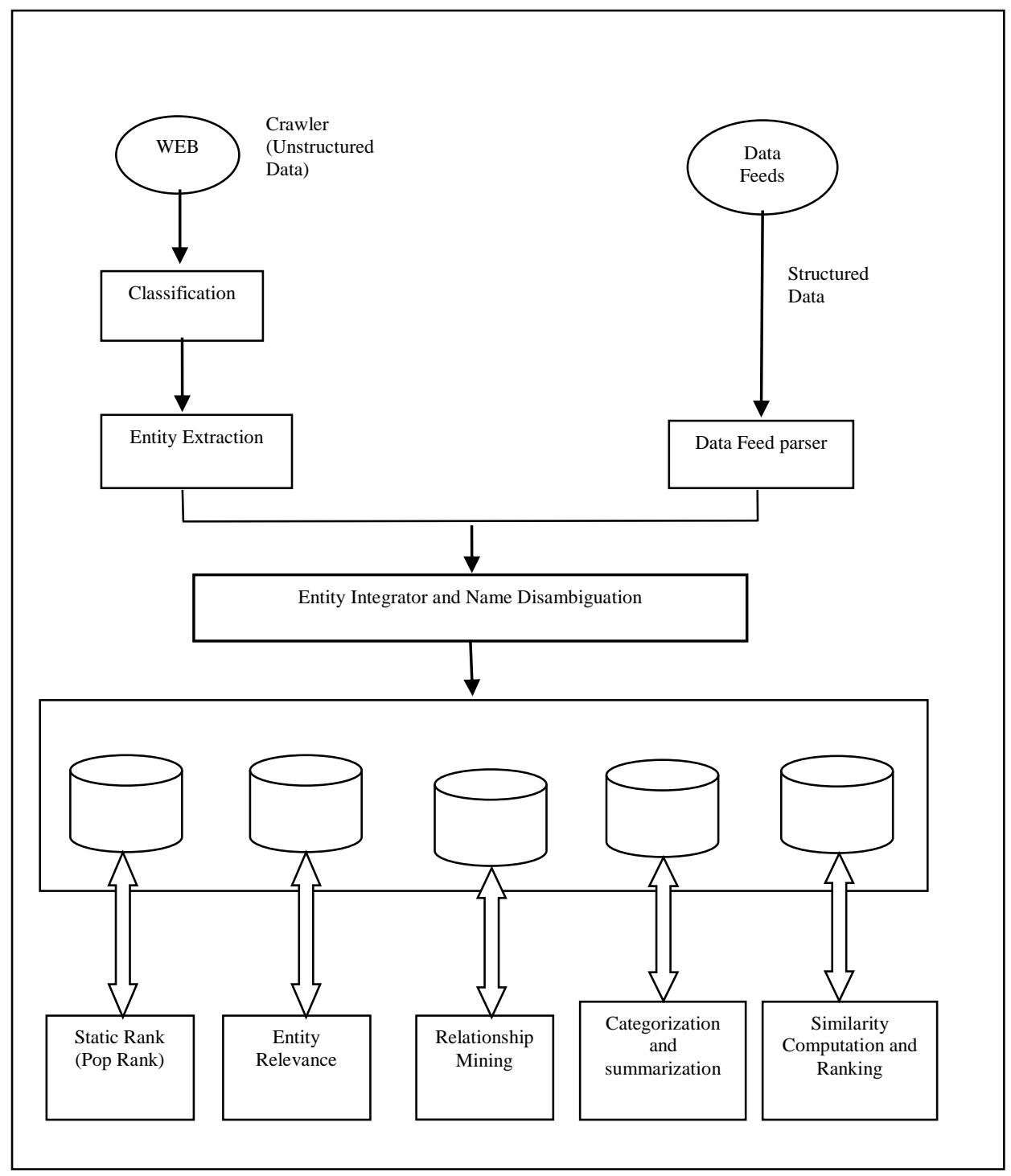

Figure 1. General Architecture of Entity Search Engine

Tao Cheng et al. [6] have proposed an offline fully automated data-driven algorithm called as Identifying Normalization Entity Synonym. This algorithm mines queries where a variety of keywords have been used to refer to the same web pages and generates an expanded set of equivalent string called entity synonyms for each keyword. This architecture comprises of three modules: Candidate generation, Candidate Selection and noise cleaning. This algorithm works well for structured data and covers the structured web queries with good precision. However, this technique is not applicable to dynamic and unstructured data.

Hamid Mousavi et al. [7] proposed a new technique to generate context aware synonyms for the entities and attributes. They proposed the Context-aware synonym Suggestion System (CS3) which learns synonyms from text by using NLP-based text mining technique called SemScape. The architecture comprises of four parts:

- Integrating existing knowledge bases by converting them into a common internal representation and storing the integrated one in another knowledge store called as Integrated Knowledge Base (IKB) store. 
- Further addition of facts from free text to IKB store.

- Large-corpus generation of context-aware synonyms that can be used to remove inconsistencies in IKB store.

- Incompleteness resolution in IKB store by using the synonyms generated in above step.

The main disadvantage of the proposal is its static approach. The knowledge bases are bound to change over time and need to be refreshed for fresh synonyms. Moreover, the approach does not tackle dynamic nature of web.

L. Jiang et al. [8] have proposed GRIAS, a Graph-based framework for discovering entity aliases motivated by the entity relationships collected from both the structured and unstructured data. The goal of this paper is to accurately identify entity aliases, especially the long tail ones from the unstructured data. The graph-based similarity is calculated between an entity and its alias candidates chosen by candidate selection method using entity relationship graph. The approach is quite static.

Tao Cheng et al. [9] have proposed an offline data-driven bottom up approach that mines query logs. They have made a fully automated solution that works on structured data to find reference entity synonyms for various entities. To achieve this, they have used a multi-step data driven approach that relies on query and click logs. The solution works by retrieving relevant web page URLs that can act as good representatives of the entity. Then, by following the URL-query click graph, they identified a list of URLs from the URLs identified earlier. The query words corresponding to this URL list will act as true synonyms by inspecting click patterns and click volume on a large subset of such urls. But, since this approach is offline and structured, it cannot handle unstructured and dynamic data evolving over web.

Surajit Chaudhuri et al. [10] have created large reference entity tables, based upon the concept initially proposed by [11], by mining entity variant from different documents. An input string is matched with the references available in reference table. If a match is found, the content of the table serve as entity synonyms.

Yanen Li et al. [12] have proposed a comprehensive approach towards entity resolution based upon syntactic patterns, query entity clicks and distribution similarity. They have focused in the fact that an entity synonym must comply with the associated context. The approach is offline and based upon the clustering model. The merit of the system is its ability to handle the heterogeneity but the scheme suffers from the drawback of static approach.

Kaushik Chakrabarti et al. [13] have proposed a general framework for discovering entity synonym based upon two novel similarity measures called as Psuedo Document Similarity and Query Context Similarity to create a set of entity synonyms. The approach is offline and dependent upon query logs.

Harada et al. [14] have proposed an approach called NEXAS (Named Entity extraction and Association Search) that finds authoritative people from web by associating a web page through identification of its real world entities. It determines nearly all relevant entities by considering top ranked web pages from SERPs. The approach is limited to people only and can easily be better substituted through references like Wikipedia. Similar arguments can be given for the work done by Kalashnikov et al. [15] and Bollegala et al. [16].

Xiang Ren et al. [17] have proposed a general heterogeneous graph-based data model that encodes problem of structured view of each entity by capturing three key concepts Synonym candidate, Web page, Keyword and different types of interactions between them. They proposed a graph based approach for ranking entity synonyms and demonstrated a closed-form optimal solution for outputting entity synonyms scores. They adopted structured view of each entity by taking into consideration other important 
structured attributes along with string name. They make use of sub-queries, tailed synonyms and tailed web pages for harvesting more synonyms. The approach is offline and suffers from a priori the requirement of candidate synonyms.

Roi Blanco et al. [18] have proposed a recommendation engine named as Spark that links initial query of user to an entity within knowledge base. It provides ranking of the related entities. The proposed system extracts several signals from a variety of data sources that includes user sessions, twitter and flicker using various cluster of computers running Hadoop. The extracted signals are combined with a machine learned ranking model that produces final recommendation of entities to user queries. Spark has been currently used in Yahoo search result pages.

Srikantiah et al. [19] have proposed a mechanism to find the synonyms from the web on the basis of inbound anchor text. They have used Search Engine Result Pages (SERPs) to find candidate synonyms of individual keywords. The technique is scalable and can be applied to dynamic, domain independent data of unstructured web. The synonyms in their case are not entity synonyms. But can be adapted to find out the entity synonyms. Their work has been a motivation for the work proposed in this paper.

Most of the above approaches are offline and static, thus cannot cater to the need of dynamically expanding web. The approach presented in this paper, uses the dynamic context created through anchor text, context and trailing part of the dynamically generated URL. The subsequent section defines the problem and the objectives to be fulfilled by the proposed work.

\section{Problem Definition and Objectives}

The proposed work aims to generate a rich-set of entity synonyms for a given entity string, says, through online dynamic approach using the anchor text, context and trailing part of URL; and to rank the synonyms based on co-page count basis.

The objectives to be achieved by the proposed approach are:

- To create an automated mechanism for generating synonyms that can be used for auto-suggestion, auto-replacement, query expansion etc.

- The method should be usable for commonly used entities in addition to well-known entities.

- The process should be dynamic and online.

- The entity synonyms set should be rich as compared to the previous approaches.

\section{Proposed Approach for Entity Synonyms Discovery}

The proposed work involves the usage of Search Engine Result Pages (SERPs) for extracting candidate entity synonyms, combines contexts with the input entity string thereafter uses anchor text of the downloaded web pages and the trailing part of the sub parent URLs to create a set of entity synonyms. The whole work of extracting synonyms is dividing into two parts

- Extraction of candidate entity synonyms

- Ranking of these synonyms based on page-count measure

The algorithm first uses the input entity to generate SERPs and URLs corresponding to those pages are collected in a list of parent URLs (PUs). Next, these parent URLs are further treated as input to generate sub parent URLs (SPUs). Thereafter, SPUs are visited one by one and downloaded web pages are retrieved in form of child documents. All pairs 
(anchor text, link) contained in child documents are collected in a hash map of anchor text and its corresponding URL as a set of child URLs. The child URLs contained in the hash map are compared with the parent URLs. If there is match between child URL and parent URL, then anchor text corresponding to child URL will act as a candidate entity synonym. The child documents are also used to find the context for input entity. The context used by the algorithm is retrieved using title and snippet of child documents. Context obtained are combined with the original entity (query string) to produce another set of candidate entity synonyms. Sub parent URLs are also compared with parent URLs, if match occurs then the trailing part of sub parent URL will act as a candidate entity synonyms.

Thus, entity synonym is extracted using three things:

- From child map in case of match

- From trailing part of sub parent url

- From combination of query and context obtained from child documents

The detailed process is shown through a flowchart as shown in Figure 2.

The page count of an entity $\mathrm{E}$ is an estimate of the number of pages that contain the entity sting $\mathrm{E}$. The candidate entity synonyms are ranked using similarity measure which in turn uses page counts of E only, candidate synonym ci only and both E, ci. A similarity measure or similarity function is a real-valued function that quantifies the similarity between two objects. It represents the similarity between two objects or words.

Let NE be the page count corresponding to input entity E only, Nci be the page counts for a candidate synonym ci only and NEci be the page counts for both entity string E and a candidate synonym ci. The proposed works uses WebJaccard similarity measure to accurately measure the relevance between $\mathrm{E}$ and ci, which is further used to rank the candidate synonyms using page counts. 


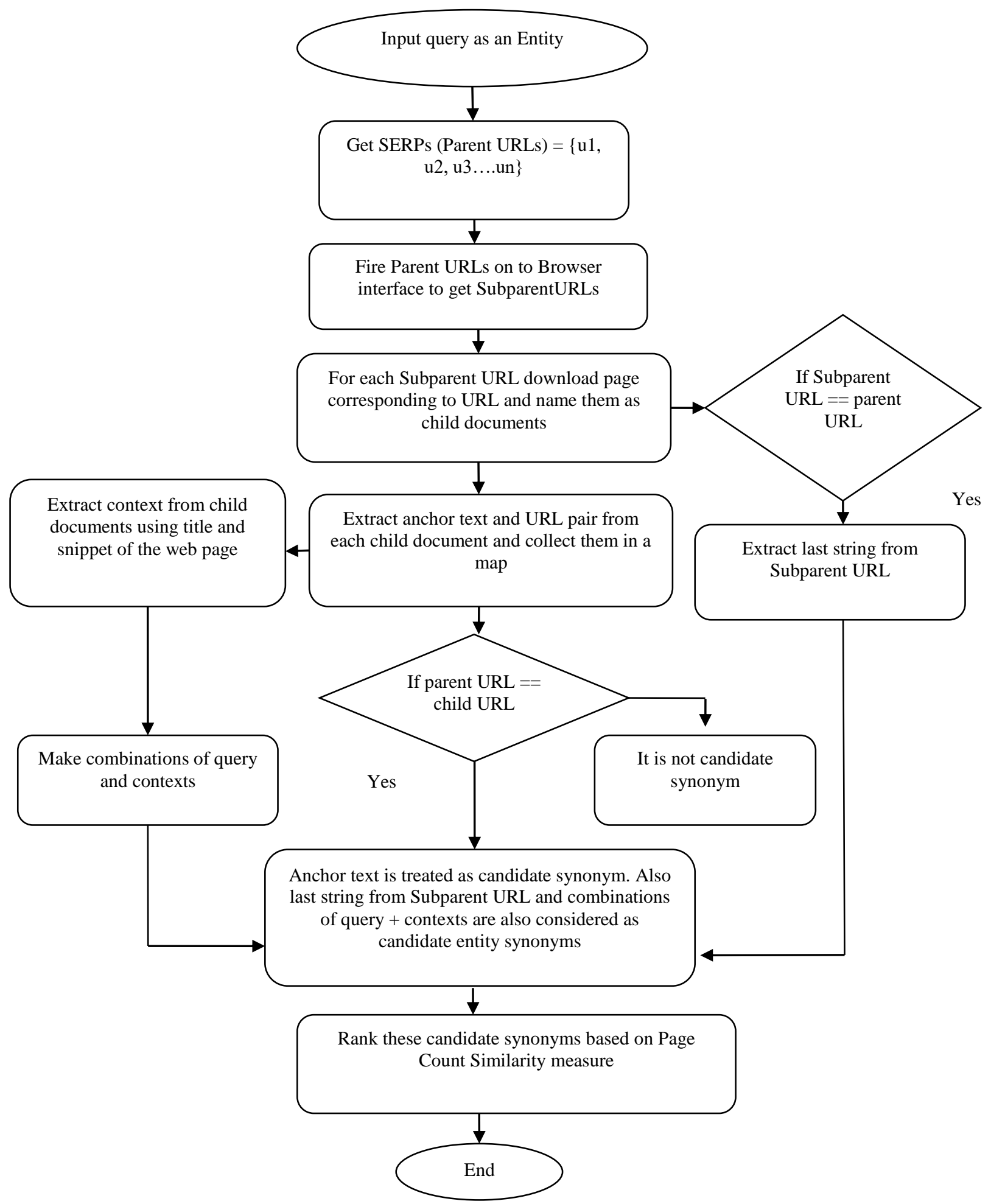

Figure 2. Flowchart of Proposed Algorithm

The details of the process have been described through the following algorithm.

\section{Algorithm (E, CS)}

Input: Entity String E.

Output: List of Candidate Synonyms for E ranked using page count measures

//Generation of Entity Candidate Synonyms 
1. Submit the input query E on to the search interface to get Search Engine Result Pages (SREPs) from search engine.

1.1. Extract all Urls from SERPs obtained above into a list of parent Urls (i.e PU)

2. For each URL $u_{j} \in P U$ do

2.1. Get all pages that contain URL $u_{j}$ into another list called as sub parent urls.

Thus, for each $u_{j}$ there will be a list called as $S P U_{j}$.

2.2. Sub parent list $(\mathrm{SPU})=\cup_{j} S P U_{j}$

3. For each URL $s u_{k} \epsilon S P U$ do

3.1. childDocument $_{\mathrm{k}}=$ Downlaod the web page corresponding to URL $s u_{k}$

3.2. childDocuments $=\cup_{k}$ childDocuments $k$

4. For each childDocument $_{i} \epsilon$ childDocuments do

4.1. Collect all anchor text and URL pair $\left(\mathrm{u}_{\mathrm{j}}, \mathrm{a}_{\mathrm{j}}\right)$ from childDocument $t_{j}$ into a hash map (i.e $\mathrm{CM}_{\mathrm{j}}$ )

4.2. Make union of all the pair collected above into a single map of anchor text and its corresponding URL. (i.e $\mathrm{CM}$ (child map) $=\mathrm{U}_{\mathrm{j}} \mathrm{CM}_{\mathrm{j}}$ )

4.3. Also extract context from each child document and collect them into a list of contexts. Context of the web page is extracted using title and snippet of the web page.

5. For each $c i \epsilon$ Context do

5.1. Combine the initial entity string $\mathrm{E}$ with context $c i$

5.2. Maintain the above combination of entity string and context into a list called as QC(Query context)

6. For each $u_{j} \in P U$ do

For each URL $s u_{k} \in S P U$ do

If $\left(u_{j}==s u_{k}\right)$ then

6.1. trailingPartofURL $=$ Extract last string from subparent url

6.2. Collect all strings obtained into a list of synonym obtained from trailing part of url called as TPU

7. For each url $u_{i} \in P U$ do

For each <url, anchortext> pair $u_{j} a_{j} \epsilon C M$ do

If $\left(u_{i}==u_{j}\right)$ then

7.1. Collect the anchor text associated with url $u_{j}$ into a list of Candidate Synonyms (CS)

8. Union synonyms obtained from child documents, <query + context> and trailing part of sub parent url into one list of candidate synonyms

8.1. Thus, $\mathrm{CS}=\cup \mathrm{TPU} \cup \mathrm{QC}$

9. //Ranking of Entity candidate synonyms

Retrieve the page counts for $E(N E)$

For each candidate synonym $c i \in C S$ do

Retrieve the page counts for $E$ and $c i(N E c i)$

Retrieve the page counts for $c i(\mathrm{Nci})$

Compute WebJaccard $(E, c i)=N E c i /(N E+N c i-N E c i)$

End for 
Table 1 shows the results of the proposed approach and its comparison with [19].

Table 1. Comparison Table

\begin{tabular}{|c|c|c|c|c|c|c|}
\hline $\begin{array}{l}\text { Sr. } \\
\text { No } \\
\text {. } \\
\end{array}$ & Entity & [19] Results & SI & $\begin{array}{l}\text { Results of Proposed } \\
\text { Algorithm }\end{array}$ & SI & $\begin{array}{l}\text { List of Context used in } \\
\text { Proposed Methodology }\end{array}$ \\
\hline 1 & inception & $\begin{array}{l}\text { Inception - } \\
\text { Wikipedia }\end{array}$ & 0.013 & inception will & 1.000 & \multirow{11}{*}{$\begin{array}{l}\text { tv, arrival, wiki, } \\
\text { overuse, while, title, - } \\
\text { LRB-, imdb, } \\
\text { inception/nostalgia, } \\
\text { will, cillian, film, } \\
\text { premiere, spot, } \\
\text { Christopher }\end{array}$} \\
\hline & & & & inception title & 0.525 & \\
\hline & & & & inception arrival & 0.395 & \\
\hline & & & & inception spot & 0.294 & \\
\hline & & & & inception tv & 0.275 & \\
\hline & & & & inception film & 0.237 & \\
\hline & & & & inception Christopher & 0.142 & \\
\hline & & & & Inception (2010) - IMDb & 0.049 & \\
\hline & & & & inception overuse & 0.018 & \\
\hline & & & & inception wiki & 0.013 & \\
\hline & & & & Inception - Wikipedia & 0.013 & \\
\hline \multirow[t]{11}{*}{2} & Nobel prize & $\begin{array}{l}\text { "The Nobel } \\
\text { Prize in } \\
\text { Physics" }\end{array}$ & 0.048 & nobel-prize & 0.984 & \multirow{11}{*}{$\begin{array}{l}\text { year, ernst, } \\
\text { chandrasekhara, } \\
\text { foundation, saal, prize, } \\
\text { norwegian, riksbank, } \\
\text { assembly, venkata, } \\
\text { winners, karl, maathaus, } \\
\text { parody, prize, curie, } \\
\text { prizes, peace, sverige, } \\
\text { step, Einstein }\end{array}$} \\
\hline & & & & nobel prize winners & 0.759 & \\
\hline & & & & nobel prize foundation & 0.166 & \\
\hline & & & & $\begin{array}{l}\text { "The Nobel Prize in } \\
\text { Physics" }\end{array}$ & 0.048 & \\
\hline & & & & nobel prize peace & 0.032 & \\
\hline & & & & nobel prize assembly & 0.019 & \\
\hline & & & & nobel prize Einstein & 0.018 & \\
\hline & & & & nobel_prize_in_physics & 0.014 & \\
\hline & & & & nobel prize curie & 0.013 & \\
\hline & & & & nobel_prize & 0.007 & \\
\hline & & & & "Nobel Prize | award" & 0.000 & \\
\hline \multirow[t]{5}{*}{3} & Bard of Avon & $\begin{array}{l}\text { The Bard of } \\
\text { Avon, William } \\
\text { Shakespeare }\end{array}$ & 0.054 & Bard of Avon shakespeare & 0.896 & \multirow{5}{*}{ shakespeare, definition } \\
\hline & & & & $\begin{array}{l}\text { why-is-shakespeare-called- } \\
\text { the-the-bard-of-avon }\end{array}$ & 0.826 & \\
\hline & & & & $\begin{array}{l}\text { bard-of-avon-the-story-of- } \\
\text { william-shakespeare }\end{array}$ & 0.806 & \\
\hline & & & & bard--of-avon & 0.089 & \\
\hline & & & & $\begin{array}{l}\text { The Bard of Avon, William } \\
\text { Shakespeare }\end{array}$ & 0.054 & \\
\hline \multirow[t]{3}{*}{4} & onocrotalus & HBW Alive & 0.006 & onocrotalus pelecanus & 0.895 & \multirow{3}{*}{$\begin{array}{l}\text { pelecanus, factsheet, } \\
\text { pelican, download, base }\end{array}$} \\
\hline & & & & pelecanus_onocrotalus & 0.797 & \\
\hline & & & & onocrotalus download & 0.643 & \\
\hline
\end{tabular}




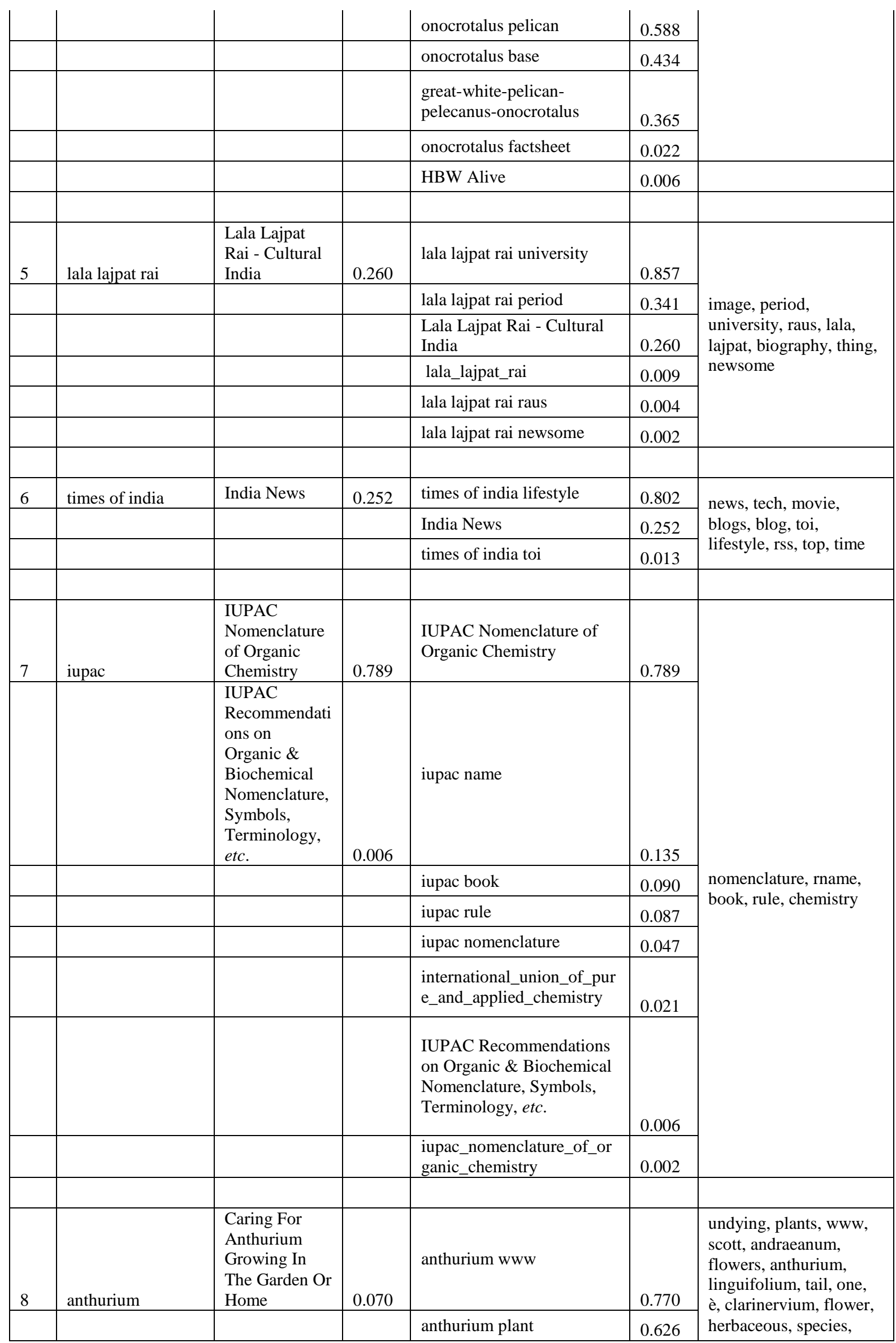




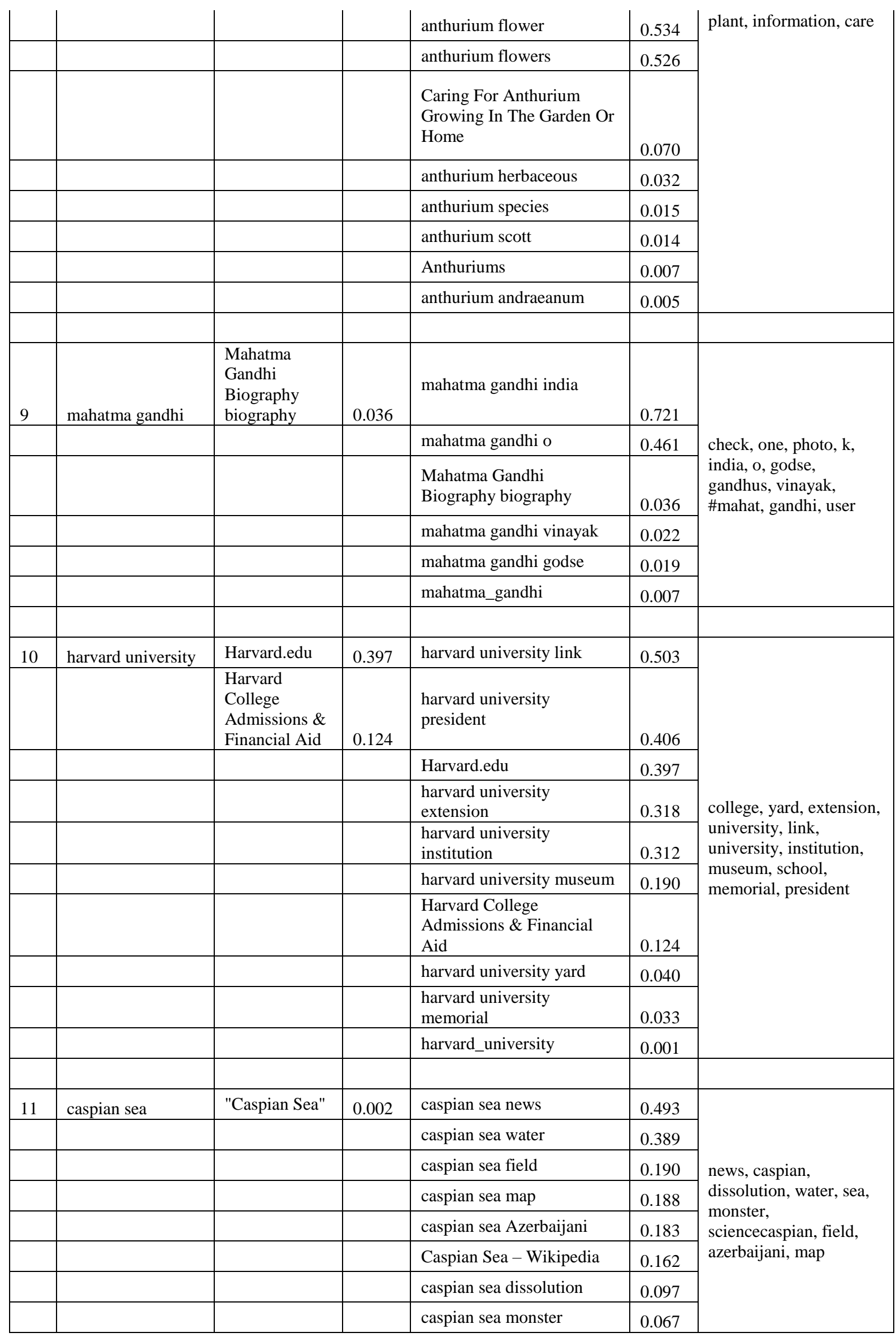




\begin{tabular}{|c|c|c|c|c|c|c|}
\hline & & & & $\begin{array}{l}\text { Caspian Sea Map, Caspian } \\
\text { Sea Location Facts History, } \\
\text { Major ... }\end{array}$ & 0.017 & \\
\hline & & & & $\begin{array}{l}\text { Caspian Sea: Largest } \\
\text { Inland Body of Water }\end{array}$ & 0.012 & \\
\hline & & & & caspian_sea & 0.011 & \\
\hline & & & & "Caspian Sea" & 0.002 & \\
\hline \multirow[t]{9}{*}{12} & moment of inertia & $\begin{array}{l}\text { Lecture notes } \\
\text { on rigid-body } \\
\text { rotation and } \\
\text { moments of } \\
\text { inertia }\end{array}$ & 0.008 & $\begin{array}{l}\text { moment of inertia } \\
\text { expression }\end{array}$ & 0.482 & \multirow{9}{*}{$\begin{array}{l}\text { expression, video, } \\
\text { moment, inertia, sewer, } \\
\text { physics, cylinder, pipe }\end{array}$} \\
\hline & & & & more-on-moment-of-inertia & 0.126 & \\
\hline & & & & moment of inertia video & 0.098 & \\
\hline & & & & $\begin{array}{l}\text { calculating-moment-of- } \\
\text { inertia-of-point-masses }\end{array}$ & 0.050 & \\
\hline & & & & moment of inertia sewer & 0.043 & \\
\hline & & & & moment of inertia physics & 0.040 & \\
\hline & & & & moment of inertia cylinder & 0.040 & \\
\hline & & & & moment of inertia pipe & 0.037 & \\
\hline & & & & $\begin{array}{l}\text { Lecture notes on rigid-body } \\
\text { rotation and moments of } \\
\text { inertia }\end{array}$ & 0.008 & \\
\hline \multirow[t]{6}{*}{13} & $\begin{array}{l}\text { jobs in computer } \\
\text { engineering }\end{array}$ & $\begin{array}{l}\text { Computer } \\
\text { Engineer }\end{array}$ & 0.384 & $\begin{array}{l}\text { jobs in computer } \\
\text { engineering diploma }\end{array}$ & 0.962 & \multirow{6}{*}{ computer, diploma } \\
\hline & & & & $\begin{array}{l}\text { computer-science- } \\
\text { engineering-jobs }\end{array}$ & 0.921 & \\
\hline & & & & computer-engineer-jobs & 0.474 & \\
\hline & & & & Computer Engineer & 0.384 & \\
\hline & & & & computer-engineering & 0.365 & \\
\hline & & & & $\begin{array}{l}\text { Computer Science } \\
\text { Engineering }\end{array}$ & 0.152 & \\
\hline \multirow[t]{7}{*}{14} & lokmanya & $\begin{array}{l}\text { Bal Gangadhar } \\
\text { Tilak }\end{array}$ & 0.067 & lokmanya www & 0.450 & \multirow{7}{*}{$\begin{array}{l}\text { coimbatore, movie, yug, } \\
\text { bal, search, bank, } \\
\text { society, tilak, www, } \\
\text { lokmanyatilak, } \\
\text { pronunciation, ek, } \\
\text { gangadhar }\end{array}$} \\
\hline & & & & lokmanya society & 0.176 & \\
\hline & & & & lokmanya tilak & 0.171 & \\
\hline & & & & lokmanya Coimbatore & 0.165 & \\
\hline & & & & lokmanya gangadhar & 0.124 & \\
\hline & & & & Bal Gangadhar Tilak & 0.067 & \\
\hline & & & & bal_gangadhar_tilak & 0.033 & \\
\hline 15 & ddlj & $\begin{array}{l}\text { Dilwale } \\
\text { Dulhania Le } \\
\text { Jayenge }\end{array}$ & 0.377 & $\begin{array}{l}\text { Dilwale Dulhania Le } \\
\text { Jayenge }\end{array}$ & 0.378 & initialism \\
\hline
\end{tabular}


International Journal of Future Generation Communication and Networking

Vol. 10, No. 11 (2017)

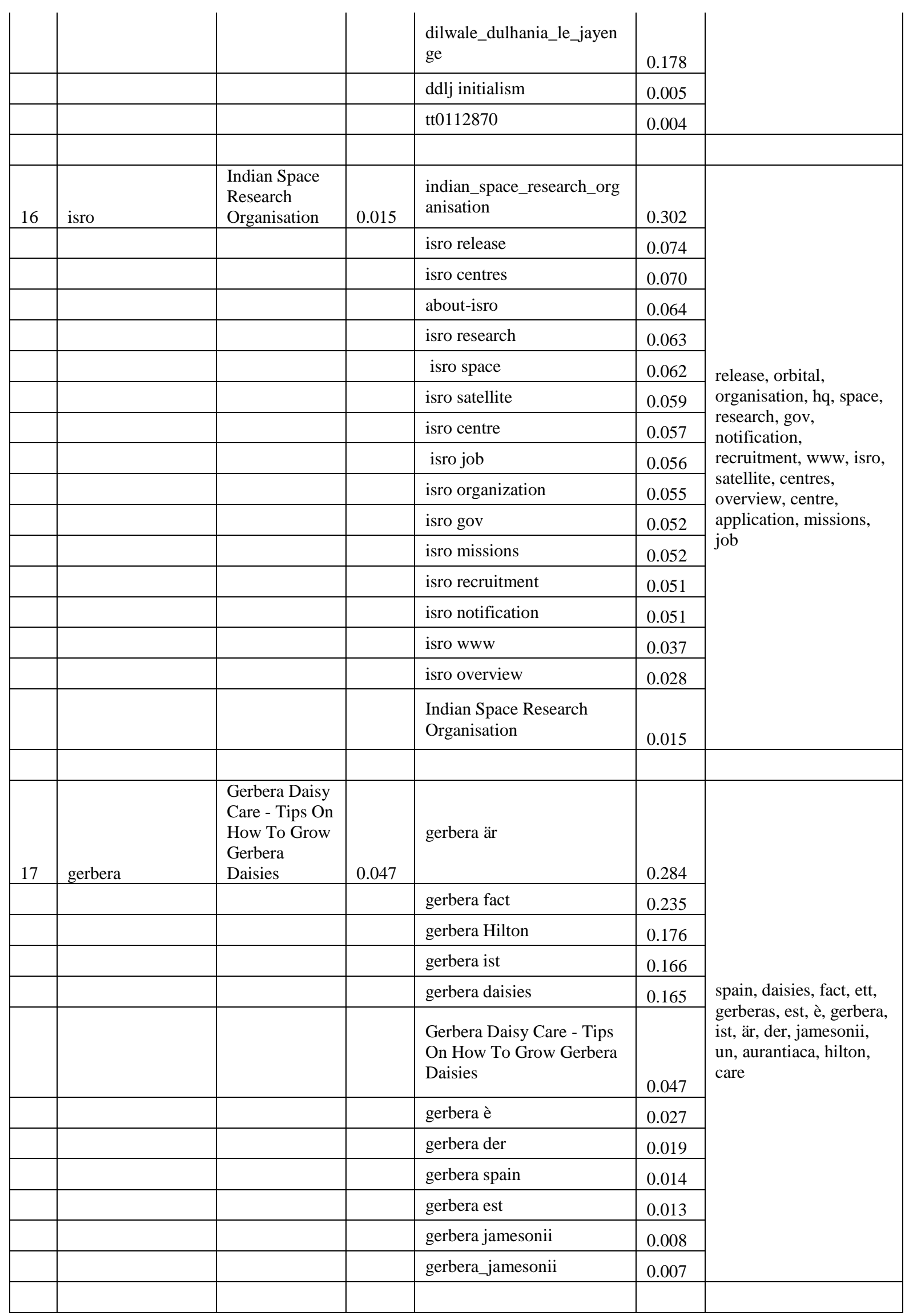




\begin{tabular}{|c|c|c|c|c|c|c|}
\hline 18 & red fort of india & $\begin{array}{l}\text { history behind } \\
\text { red fort history } \\
\text { of india }\end{array}$ & 0.062 & red fort of india moti & 0.185 & \multirow{5}{*}{ moti, shahjahan } \\
\hline & & $\begin{array}{l}\text { Red Fort - } \\
\text { Wikipedia, the } \\
\text { free } \\
\text { encyclopedia }\end{array}$ & 0.021 & $\begin{array}{l}\text { HISTORY BEHIND RED } \\
\text { FORT HISTORY OF } \\
\text { INDIA }\end{array}$ & 0.062 & \\
\hline & & & & red fort of india shahjahan & 0.029 & \\
\hline & & & & $\begin{array}{l}\text { Red Fort - Wikipedia, the } \\
\text { free encyclopedia }\end{array}$ & 0.021 & \\
\hline & & & & red_fort & 0.018 & \\
\hline \multirow[t]{9}{*}{19} & vivekanand & $\begin{array}{l}\text { The Life and } \\
\text { Teachings of } \\
\text { Swami } \\
\text { Vivekananda }\end{array}$ & 0.031 & vivekanand life & 0.157 & \multirow{9}{*}{$\begin{array}{l}\text { biographical, } \\
\text { bibliography, thought, } \\
\text { swami, teachings, life }\end{array}$} \\
\hline & & & & $\begin{array}{l}\text { The Life and Teachings of } \\
\text { Swami Vivekananda }\end{array}$ & 0.031 & \\
\hline & & & & vivekanand teachings & 0.030 & \\
\hline & & & & vivekanand swami & 0.026 & \\
\hline & & & & vivekanand biographical & 0.025 & \\
\hline & & & & vivekanand thought & 0.023 & \\
\hline & & & & swami_vivekananda & 0.021 & \\
\hline & & & & vivekanand bibliography & 0.019 & \\
\hline & & & & $\begin{array}{l}\text { stories-swami- } \\
\text { vivekananda-life-inspired }\end{array}$ & 0.010 & \\
\hline \multirow[t]{3}{*}{20} & $\begin{array}{l}\text { terms used in } \\
\text { biology }\end{array}$ & $\begin{array}{l}\text { Glossary of } \\
\text { biology - } \\
\text { Wikipedia } \\
\end{array}$ & 0.150 & $\begin{array}{l}\text { Glossary of biology - } \\
\text { Wikipedia }\end{array}$ & 0.150 & \\
\hline & & $\begin{array}{l}\text { Biology } \\
\text { Vocabulary: } \\
\text { Understanding } \\
\text { Common } \\
\text { Terms } \\
\end{array}$ & 0.010 & biology-vocabulary & 0.055 & \\
\hline & & & & $\begin{array}{l}\text { Biology Vocabulary: } \\
\text { Understanding Common } \\
\text { Terms }\end{array}$ & 0.010 & \\
\hline
\end{tabular}

\section{Using the Results}

The hallmark of proposed scheme is that it not only computes a rich-set of entity synonyms, but also shows the extent of matching with the original entity string. This known extent of matching can be used in the same manner as used in case of alpha-cut in case of fuzzy logic. The designer of the search engine can make decisions about the cut of values for using a synonym for a particular purpose. For example, the lower cut off value for auto-suggestion, auto-replacement and query expansion can be 0.6, 0.75 and 0.9 respectively. The ranks can also be fuzzified by designing the appropriate fuzzy-sets which can be used for automating the query auto-suggestion, replacement and expansion processes through a system like fuzzy-rule based inference system. 


\section{Conclusion and Future Scope}

The proposed work is able to generate a comparatively richer set of entity synonyms than its predecessors and is able to assess the quality of the synonyms as well. The work can be further refined by the augmentation of synonyms retrieved from query log. The relationship between the entity string and the corresponding synonyms can be exhibited using an entity graph through which various possibilities can be explored including the machine intelligence.

\section{References}

[1] M. M. Stark and R. F. Riesenfeld, "Wordnet: An electronic lexical database", In Proceedings of 11th Eurographics Workshop on Rendering, MIT Press, 1998J. Breckling, Edition, The Analysis of Directional Time Series: Applications to Wind Speed and Direction, ser. Lecture Notes in Statistics. Berlin, Germany: Springer, vol. 61, (1989).

[2] G. K. Ralf Schenkel and Fabian Suchanek, "Yawn: A semantically annotated wikipedia xml corpus", In GI-Fachtagung fur Datenbanksysteme in Business, Technologie und Web, volume 103 of LNI, (2007).

[3] K. D. Bollacker, C. Evans, P. Paritosh, T. Sturge and J. Taylor, "Freebase:a collaboratively created graph database for structuring human knowledge", In SIGMOD, (2008).

[4] P. Paul and T. George, "Entity Search Engines", In International Journal of Computer Science and Mobile Computing, vol.3, iss. 2, (2014), pg. 877-880

[5] S. Chaudhuri, V. Ganti and D. Xin, "Exploiting Web Search to Generate Synonyms for Entities", In WWW '09 Proceedings of the 18th international conference on World wide web, Madrid, Spain, (2009).

[6] T. Cheng, H. W. Lauw and S. Paparizos, "Entity Synonyms for Structured Web Search", IEEE Transactions on Knowledge and Data Engineering, vol. 24, no. 10, (2012), pp. 1862-1873.

[7] H. Mousavi, S. Gao and C. Zaniolo, "Discovering Attribute and Entity Synonyms for Knowledge Integration and Semantic Web Search", In Proceedings of the 3rd International Workshop on Semantic Search Over the Web, Riva del Garda, Italy, (2013).

[8] L. Jiang, P. Luo, J. Wang, Y. Xiong, B. Lin, M. Wang and N. An. Grias, “An entity-relation graph based framework for discovering entity aliases", In ICDM, (2013).

[9] T. Cheng, H. W. Lauw and S. Paparizos, "Fuzzy Matching of Web Queries to Structured Data", In Data Engineering (ICDE), 2010 IEEE 26th International Conference, (2010).

[10] S. Chaudhuri, V. Ganti and D. Xin, "Mining Document Collections to Facilitate Accurate Approximate Entity Matching", In PVLDB, (2009).

[11] A. Aho and M. Corasick, "Efficient string matching: an aid to bibliographic search", In Comm. ACM, vol. 18, (1975).

[12] Y. Li, B.-J. (Paul) Hsu, C.X. Zhai and K. Wang, "Mining Entity Attribute Synonyms via Compact Clustering", In CIKM '13 Proceedings of the 22nd ACM international conference on Information \& Knowledge Management, San Francisco, California, USA, (2013).

[13] K. Chakrabarti, S. Chaudhuri, T. Cheng and D. Xin, "A Framework for Robust Discovery of Entity Synonyms", In KDD '12 Proceedings of the 18th ACM SIGKDD international conference on Knowledge discovery and data mining, Beijing, China, (2012).

[14] M. Harada, S.-Y. Sato and K. Kazama, "Finding Authoritative People from the Web", In Proceedings of the Joint ACM/IEEE Conference on Digital Libraries (JCDL04), (2004), pp. 306-313.

[15] K. Dmitri, V. Z. (Stella) Chen, S. Mehrotra and R. Nuray-Turan, "Web People Search via Connection Analysis", IEEE Transactions on Knowledge and Data Engineering, vol. 20, no. 11, (2008), pp. 1-16.

[16] D. Bollegala, Y. Matsuo and M. Ishizuka, "Automatic Discovery of Personal Name Aliases from the Web", IEEE Transactions on Knowledge and Data Engineering, vol. 23, no. 6, (2011), pp. 831-844.

[17] X. Ren and T. Cheng, "Synonym Discovery for Structured Entities on Heterogeneous Graphs", In WWW '15 Companion Proceedings of the 24th International Conference on World Wide Web, Florence, Italy, (2015).

[18] R. Blanco, B. Barla Cambazoglu1, P. Mika and N. Torzec, "Entity Recommendations in Web Search", In International Semantic Web Conference, (2013).

[19] K. C. Srikantiah, M. S. Roopa, N. Krishna Kumar, V. Tejaswi, K. R. Venugopal and L. M. Patnaik, "Automatic Discovery of Synonyms from the Web based on Inbound Anchor Text", ICDMW, () Elsevier Publications, pp. 130-139. 


\section{Authors}

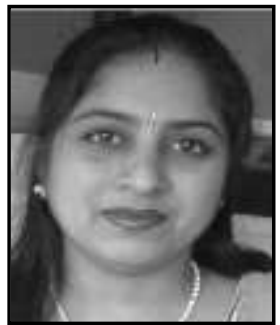

Mamta Kathuria, she received her MCA degree from Kurukshetra University and M.Tech from MDU, Rohtak in 2008, respectively.. She is pursuing her Ph.D in Computer Engineering from YMCA University of Science and Technology, Faridabad. She is currently working as a Assistant Professor in YMCA University of Science \& Technology and has eight years of experience. Her areas of interest are search engines, Web Mining and Fuzzy Logic.

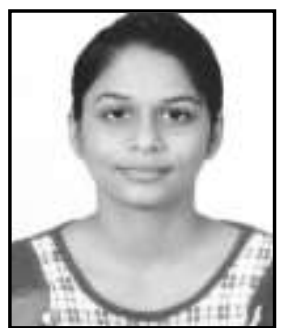

Anuradha, she received B. Tech in Computer Engineering from YMCA university of Science \& Engineering, Faridabad in 2014. She is pursuing her $\mathrm{M}$. Tech in Computer Engineering from YMCA University of Science and Technology, Faridabad. Her areas of interest are Search Engines, Information Retrieval and Enhancing the quality of Web Search.

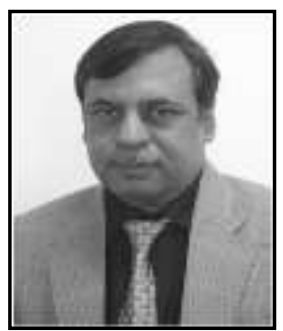

Chander K. Nagpal, he is $\mathrm{Ph}$. D (CS) from Jamia Milla Islamia, New Delhi. He is currently working as a professor in YMCA University of Science \& Technology and has twenty eight years of teaching experience. He has published two books. He has published many research papers in reputed international Journals such as IEEE transaction, Wiley STVR, CSI. His academic interests include Ad hoc networks, Web Mining and Soft Computing.

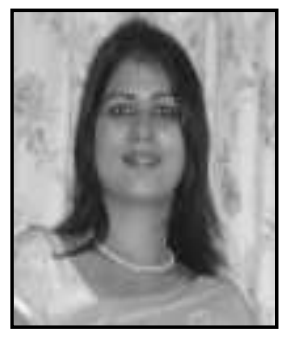

Neelam Duhan, she received M. Tech in Computer Engineering from MDU, Rohtak in 2005. She completed her PhD in (CE) in 2011 from Maharshi Dayanand University, Rohtak. She is currently working as an Assistant Professor in CE Department in YMCA University of Science and Technology, Faridabad and has an experience of about 12 years. She has published over 30 research papers in reputed international Journals and Conferences. Her areas of interest are databases, search engines and web mining. 
International Journal of Future Generation Communication and Networking Vol. 10, No. 11 (2017) 